\title{
Iterative Identification Algorithms for Continuous-Time Systems with Unknown Time Delay in the Presence of Measurement Noise
}

\author{
Zi-Jiang Yang* Member \\ Hideto Iemura* Non-member \\ Shunshoku Kanae* Non-member \\ Kiyoshi Wada* Member
}

\begin{abstract}
This paper considers the identification problem of continuous-time systems with unknown time delay from sampled input-output data. By using a digital pre-filter, an approximated discrete-time estimation model is first derived, in which the system parameters remain in their original form and the time delay need not be an integral multiple of the sampling period. Then an unseparable nonlinear least-squares (UNSEPNLS) method and a separable nonlinear least-squares (SEPNLS) method for identification of transfer function parameters and time delay are derived. Furthermore, an unseparable nonlinear instrumental variable (UNSEPNIV) method and a separable nonlinear instrumental variable (SEPNIV) method are proposed, to eliminate estimate bias due to measurement noise. Simulational results show that the UNSEPNIV and SEPNIV methods yield consistent estimates in the presence of measurement noise.
\end{abstract}

Keywords: Time delay, continuous-time system, identification, nonlinear least-squares method, instrumental variable method, iterative algorithm.

\section{Introduction}

Many practical systems such as thermal processes, chemical processes and biological systems, etc., have inherent time delay. Therefore, the problem of identifying such a system is of great importance for analysis, synthesis and prediction.

Numerous identification methods of time delay systems based on the discrete-time model have been proposed $^{(1) \sim(3)}$. In the case of the discrete-time model, the sampling period is required to be very small such that the time delay is an integral multiple of the sampling period, whereas if the sampling period is too small, the identification problem may become numerically difficult ${ }^{(4)}$.

There have been some typical approaches to identification of continuous-time models with unknown delay. One approach is based on the approximation of the time delay in the frequency-domain by a rational transfer function or the padé approximation ${ }^{(5) \sim(7)}$. This approach requires estimation of more parameters because the order of the approximated system model is increased. And it is not easy to separate the parameters concerned with the time delay from those concerned with the transfer function of the system. An other problem in this method is that it may introduce unacceptable approximation error when the system has large time delay.

Another approach is based on the nonlinear estimation method like nonlinear LS method that searches

\footnotetext{
* Graduate School of Information Science and Electrical Engineering, Kyushu University

6-10-1, Hakozaki, Higashi-ku, Fukuoka 812-8581
}

for the optimum by using a gradient-following technique ${ }^{(8) \sim(10)}$. In references (8) (10), some variations of pure continuous-time on-line nonlinear LS methods were studied. In reference (9) the off-line SEPNLS method was studied, where the time delay is searched exhaustively with spline interpolation of the LS criterion. However, consistent identification of continuous-time systems with unknown time delay in the presence of measurement noise of considerably high level has not been studied extensively in the literature.

This paper considers the identification problem of continuous-time systems with unknown time delay from sampled input-output data. An UNSEPNLS method and a SEPNLS method for identification of transfer function parameters and time delay are derived. Furthermore, an UNSEPNIV method and a SEPNIV method are proposed to eliminate estimate bias due to measurement noise. Simulational results show that the SEPNIV and UNSEPIV methods yield consistent estimates.

\section{Statement of the problem}

Consider the following single-input single-output continuous-time system with unknown time delay:

$$
\begin{array}{r}
\sum_{i=0}^{n} a_{i} p^{n-i} x(t)=\sum_{i=1}^{m} b_{i} p^{m-i} u(t-\tau) \\
\left(a_{0}=1, b_{1} \neq 0\right)
\end{array}
$$

where $p$ is a differential operator, $u(t), x(t)$ are real input and output, and $\tau$ is the time delay.

It is assumed that a zero-order hold is utilized:

$$
u(t)=\bar{u}(k) \quad(k-1) T \leq t<k T
$$


where $T$ is the sampling period.

Practically the discrete-time measurement of the output is corrupted by a stochastic measurement noise.

$$
y(k)=x(k)+v(k)
$$

where $y(k), x(k), v(k)$ denote $y(k T), x(k T), v(k T)$ respectively.

Our goal is to identify the time delay and the system parameters from sampled data of the input and the noisy output. To clarify the problem setting, some assumptions are made here.

Assumption 1: The system under study is open loop strictly stable and strictly proper with known order $n$ and relative degree $n-m+1(n \geq m)$.

Assumption 2: The input $\bar{u}(k)$ is a quasi-stationary deterministic or random signal and the noise $v(k)$ is a quasi-stationary zero-mean random signal uncorrelated with the input such that $\lim _{N \rightarrow \infty} 1 / N \sum_{k=1}^{N} \bar{u}(k) v(k)=$ 0 . This is a common assumption for the instrumental variable technique ${ }^{(11)}$.

\section{Approximated Discrete-Time Estima- tion model}

To avoid the direct signal derivatives, we introduce a low-pass pre-filter $Q(p)$ as ${ }^{(12)}$

$$
Q(p)=\frac{1}{(\alpha p+1)^{n}}
$$

where $\alpha$ is the time constant which determines the passband of $Q(p)$.

Multipling both sides of (1) by $Q(p)$ and using the bilinear transformation based on the block-pulse functions ${ }^{(13)(14)}$, we can obtain the following approximated discrete-time estimation model of the original system:

$$
\xi_{0 \bar{y}}(k)+\sum_{i=1}^{n} a_{i} \xi_{i \bar{y}}(k)=\sum_{i=1}^{m} b_{i} \xi_{(n-m+i) \bar{u}}(k-\tilde{\tau})+r(k)
$$

where

$$
r(k)=\sum_{i=0}^{n} a_{i} \xi_{i \bar{v}}(k)
$$

and

$$
\begin{aligned}
& \xi_{i \bar{u}}(k)=\frac{\left(\frac{T}{2}\right)^{i}\left(1+z^{-1}\right)^{i}\left(1-z^{-1}\right)^{n-i}}{\left[\alpha\left(1-z^{-1}\right)+\frac{T}{2}\left(1+z^{-1}\right)\right]^{n}} \bar{u}(k) \\
& \xi_{i \bar{y}}(k)=\frac{\left(\frac{T}{2}\right)^{i}\left(1+z^{-1}\right)^{i}\left(1-z^{-1}\right)^{n-i}}{\left[\alpha\left(1-z^{-1}\right)+\frac{T}{2}\left(1+z^{-1}\right)\right]^{n}} \bar{y}(k) \\
& \xi_{i \bar{v}}(k)=\frac{\left(\frac{T}{2}\right)^{i}\left(1+z^{-1}\right)^{i}\left(1-z^{-1}\right)^{n-i}}{\left[\alpha\left(1-z^{-1}\right)+\frac{T}{2}\left(1+z^{-1}\right)\right]^{n}} \bar{v}(k)
\end{aligned}
$$

where $\bar{v}(k)=\left(1+z^{-1}\right) v(k) / 2$ and $\bar{y}(k)=\left(1+z^{-1}\right) y(k) / 2$. $\tilde{\tau}$ in (5) is given by

$$
\tilde{\tau}=\tau / T=l+\Delta / T
$$

where $0 \leq \Delta<T$ and $l$ is a non-negative integer.

Remark 1: In the case where the system is excited by a band limited continuous input signal $u(t)$ instead of the piecewise-constant signal given in $(2)$, we can define $\bar{u}(k)=\left(1+z^{-1}\right) u(k) / 2$. In this case, the bilinear transformation corresponds to the trapezoidal integration law ${ }^{(14)}$.

Remark 2: Usually it is desirable that the time delay $\tau$ is an integral multiple of the sampling period, whereas our estimation model does not require this restriction. In the case of $\Delta \neq 0$, we can get $\xi_{(n-m+i) \bar{u}}(k-\tilde{\tau})$ by linear interpolation between $\xi_{(n-m+i) \bar{u}}(k-l)$ and $\xi_{(n-m+i) \bar{u}}(k-l-1)$.

(5) can be written in vector form:

$$
\begin{aligned}
\xi_{0 \bar{y}}(k)= & \boldsymbol{\varphi}^{T}(k, \tau) \boldsymbol{\theta}+r(k) \\
\boldsymbol{\varphi}^{T}(k, \tau)= & {\left[-\xi_{1 \bar{y}}(k), \cdots,-\xi_{n \bar{y}}(k),\right.} \\
& \left.\xi_{(n-m+1) \bar{u}}(k-\tilde{\tau}), \cdots, \xi_{n \bar{u}}(k-\tilde{\tau})\right] \\
\boldsymbol{\theta}^{T}= & {\left[a_{1}, \cdots, a_{n}, b_{1}, \cdots, b_{m}\right] }
\end{aligned} .
$$

\section{Nonlinear Least-Squares Methods}

Given a fixed set of filtered input-output data $\left\{\xi_{0 \bar{y}}(k), \xi_{1 \bar{y}}(k), \cdots, \xi_{n \bar{y}}(k), \xi_{(n-m+1) \bar{u}}(k), \cdots, \xi_{n \bar{u}}(k)\right\}_{k_{s}+1}^{N}$, the off-line parameter estimates are defined as the minimizing arguments of the following LS criterion

$$
\begin{aligned}
& V_{N}(\boldsymbol{\theta}, \tau)=\frac{1}{N-k_{s}} \sum_{k=k_{s}+1}^{N} \frac{1}{2} \epsilon^{2}(k, \boldsymbol{\theta}, \tau) \\
& \epsilon(k, \boldsymbol{\theta}, \tau)=\xi_{0 \bar{y}}(k)-\boldsymbol{\varphi}^{T}(k, \tau) \boldsymbol{\theta}
\end{aligned}
$$

such that

$$
\left[\widehat{\boldsymbol{\theta}}_{N}^{T}, \widehat{\tau}_{N}\right]^{T}=\arg \min _{\boldsymbol{\theta}, \tau} V_{N}(\boldsymbol{\theta}, \tau)
$$

For detailed descriptions of the iterative search algorithms applied to system identification, the readers are referred to ${ }^{(11)(15)(16)}$.

4.1 UNSEPNLS Method This method estimates $\boldsymbol{\theta}$ and $\tau$ simultaneously by minimizing the above LS criterion. Starting at an initial estimate $\widehat{\boldsymbol{\theta}}_{N}^{(0)}$ and $\widehat{\tau}_{N}^{(0)}$, the minimizing problem can be described by the following iteration (Gauss Newton method) ${ }^{(11)(15)(16)}$.

$$
\widehat{\boldsymbol{\Theta}}_{N}^{(j+1)}=\widehat{\boldsymbol{\Theta}}_{N}^{(j)}-\mu_{N}^{(j)}\left[R_{N}\left(\widehat{\boldsymbol{\Theta}}_{N}^{(j)}\right)\right]^{-1} V_{N}^{\prime}\left(\widehat{\boldsymbol{\Theta}}_{N}^{(j)}\right)
$$

where $\boldsymbol{\Theta}=\left[\boldsymbol{\theta}^{T}, \tau\right]^{T}, \mu_{N}^{(j)}$ is the step-size which assures $V_{N}$ decreases and $\widehat{\tau}_{N}$ stays in a preassigned interval, i.e., $\widehat{\tau}_{N}^{(j+1)} \in \Omega_{\tau}=\left\{\widehat{\tau}_{N}^{(j+1)} \mid 0 \leq \widehat{\tau}_{N}^{(j+1)} \leq \bar{\tau},{ }^{\exists} \bar{\tau}>0\right\} . \quad V_{N}^{\prime}(\boldsymbol{\Theta})$ and $R_{N}(\boldsymbol{\Theta})$ are respectively the gradient and the estimate of the Hessian of the LS criterion ${ }^{(11)(15)(16)}$ :

$$
\begin{aligned}
& V_{N}^{\prime}(\boldsymbol{\Theta})=-\frac{1}{N-k_{s}} \sum_{k=k_{s}+1}^{N} \boldsymbol{\zeta}(k, \boldsymbol{\Theta}) \epsilon(k, \boldsymbol{\Theta}) \\
& R_{N}(\boldsymbol{\Theta})=\frac{1}{N-k_{s}} \sum_{k=k_{s}+1}^{N} \boldsymbol{\zeta}(k, \boldsymbol{\Theta}) \boldsymbol{\zeta}^{T}(k, \boldsymbol{\Theta})
\end{aligned}
$$


where

$$
\begin{aligned}
& \boldsymbol{\zeta}(k, \boldsymbol{\Theta})=-\frac{\partial \epsilon(k, \boldsymbol{\Theta})}{\partial \boldsymbol{\Theta}} \\
& =\left[-\xi_{1 \bar{y}}(k), \cdots,-\xi_{n \bar{y}}(k), \xi_{(n-m+1) \bar{u}}(k-\tilde{\tau}),\right. \\
& \left.\cdots, \xi_{n \bar{u}}(k-\tilde{\tau}),-\sum_{i=1}^{m} b_{i} \xi_{(n-m+i-1) \bar{u}}(k-\tilde{\tau})\right]^{T}
\end{aligned}
$$

The last element of $\boldsymbol{\zeta}(k, \boldsymbol{\Theta})$, i.e., $-\partial \epsilon(k) / \partial \tau$ is derived as the following by approximating the differentiation with respect to time $t$ via bilinear transformation.

$$
\begin{aligned}
-\frac{\partial \epsilon(k)}{\partial \tau} & =\left.\sum_{i=1}^{m} b_{i} \frac{\partial}{\partial \tau} \xi_{(n-m+i) \bar{u}}(t-\tau)\right|_{t=k T} \\
& =-\left.\sum_{i=1}^{m} b_{i} \frac{d}{d t} \xi_{(n-m+i) \bar{u}}(t-\tau)\right|_{t=k T} \\
& \approx-\sum_{i=1}^{m} b_{i} \xi_{(n-m+i-1) \bar{u}}(k-\widetilde{\tau})
\end{aligned}
$$

To avoid the ill-conditioned problem, $R_{N}\left(\widehat{\boldsymbol{\Theta}}_{N}^{(j)}\right)$ should not be singular or near singular. This requires that $b_{1} \neq 0$, since if $b_{1} \approx 0$, then the last element of $\boldsymbol{\zeta}(k, \boldsymbol{\Theta})$ can be expressed by the linear combination of the other elements of $\boldsymbol{\zeta}(k, \boldsymbol{\Theta})$ such that $R_{N}\left(\widehat{\boldsymbol{\Theta}}_{N}^{(j)}\right)$ is rank defficient. To summarize, we have the following proposition:

Proposition 1 If the relative degree of system (1) is not well defined, i.e., $b_{1} \approx 0$, then the LS minimization problem with respect to $\boldsymbol{\Theta}=\left[\boldsymbol{\theta}^{T}, \tau\right]^{T}$ becomes illconditioned.

The UNSEPNLS algorithm which delivers the estimates of $\boldsymbol{\theta}$ and $\tau$ simultaneously is summarized as follows:

(1) Let $j=0$. Set the initial estimate $\widehat{\boldsymbol{\Theta}}_{N}^{(0)}$ and the considerable upper bound of time delay $\bar{\tau}$.

(2) Perform the following.

(a) Compute

$$
\Delta \widehat{\boldsymbol{\Theta}}_{N}^{(j+1)}=-\left[R_{N}\left(\widehat{\boldsymbol{\Theta}}_{N}^{(j)}\right)\right]^{-1} V_{N}^{\prime}\left(\widehat{\boldsymbol{\Theta}}_{N}^{(j)}\right)
$$

(b) Compute

$$
\widehat{\boldsymbol{\Theta}}_{N}^{(j+1)}=\widehat{\boldsymbol{\Theta}}_{N}^{(j)}+\Delta \widehat{\boldsymbol{\Theta}}_{N}^{(j+1)}
$$

(c) Check if $0 \leq \widehat{\tau}_{N}^{(j+1)} \leq \bar{\tau}$. If not, let $\Delta \widehat{\boldsymbol{\Theta}}_{N}^{(j+1)}$ $=0.5 \Delta \widehat{\boldsymbol{\Theta}}_{N}^{(j+1)}$ and go back to (b).

(d) Check if $V_{N}\left(\widehat{\boldsymbol{\Theta}}_{N}^{(j+1)}\right) \leq V_{N}\left(\widehat{\boldsymbol{\Theta}}_{N}^{(j)}\right)$. If not, let $\Delta \widehat{\boldsymbol{\Theta}}_{N}^{(j+1)}=0.5 \Delta \widehat{\boldsymbol{\Theta}}_{N}^{(j+1)}$ and go back to (b).

(3) Terminate the algorithm if the stopping condition is satisfied. Otherwise, let $j=j+1$ and go back to step 2 .

4.2 SEPNLS Method In contrast to the UNSEPNLS method, the SEPNLS method estimates the time delay $\tau$ and the linear parameters $\boldsymbol{\theta}$ in a separable manner. If the time delay is known, the linear parameters can be estimated by the linear LS method as

$$
\begin{aligned}
\widehat{\boldsymbol{\theta}}_{N}(\tau) & =\boldsymbol{R}^{-1}(N, \tau) \boldsymbol{f}(N, \tau) \\
\boldsymbol{R}(N, \tau) & =\frac{1}{N-k_{s}} \sum_{k=k_{s}+1}^{N} \boldsymbol{\varphi}(k, \tau) \boldsymbol{\varphi}^{T}(k, \tau) \\
\boldsymbol{f}(N, \tau) & =\frac{1}{N-k_{s}} \sum_{k=k_{s}+1}^{N} \varphi(k, \tau) \xi_{0 \bar{y}}(k)
\end{aligned}
$$

Then the LS criterion $V_{N}(\boldsymbol{\theta}, \tau)$ becomes the following so that the time delay can be estimated separably.

$$
\breve{V}_{N}(\tau)=\frac{1}{N-k_{s}} \sum_{k=k_{s}+1}^{N} \frac{1}{2} \breve{\varepsilon}^{2}(k, \tau) \cdots \cdots \cdots
$$

where

$$
\breve{\varepsilon}(k, \tau)=\xi_{0 \bar{y}}(k)-\varphi^{T}(k, \tau) \boldsymbol{R}^{-1}(N, \tau) \boldsymbol{f}(N, \tau) \cdots
$$

A nonlinear LS problem is called separable if one set of parameters enter linearly and another set nonlinearly in the model for parameter estimation ${ }^{(15)(17)}$. The time delay $\tau$ and the linear parameters $\boldsymbol{\theta}$ can be estimated separably according to the following theorem. See references (15) (17) for proof and more detailed explanations. Theorem 1 Let $\boldsymbol{\theta}=\widehat{\boldsymbol{\theta}}_{N}(\tau)=\boldsymbol{R}^{-1}(N, \tau) \boldsymbol{f}(N, \tau)$ denotes one solution of the LS criterion (10). Then

$$
\left[\widehat{\boldsymbol{\theta}}_{N}^{T}, \widehat{\tau}_{N}\right]^{T}=\arg \min _{\boldsymbol{\theta}, \tau} V_{N}(\boldsymbol{\theta}, \tau)=\arg \min _{\tau} \breve{V}_{N}(\tau) \cdots
$$

Since the speparable iterative research algorithm for the time delay is not found in the literature, the derivation of the algorithm will be described in detail.

The estimate of time delay can be obtained as

$$
\widehat{\tau}_{N}=\arg \min _{\tau} \breve{V}_{N}(\tau)
$$

through the following iterative search algorithm.

$$
\widehat{\tau}_{N}^{(j+1)}=\widehat{\tau}_{N}^{(j)}-\mu_{N}^{(j)}\left[\breve{R}_{N}\left(\widehat{\tau}_{N}^{(j)}\right)\right]^{-1} \breve{V}_{N}^{\prime}\left(\widehat{\tau}_{N}^{(j)}\right) \cdots
$$

where $\mu_{N}^{(j)}$ is the step-size, $\breve{V}_{N}^{\prime}(\tau)$ and $\breve{R}_{N}(\tau)$ are respectively the gradient and the estimate of the Hessian of the LS criterion (17):

$$
\begin{aligned}
\breve{V}_{N}^{\prime}(\tau) & =-\frac{1}{N-k_{s}} \sum_{k=k_{s}+1}^{N} \psi(k, \tau) \breve{\varepsilon}(k, \tau) \\
\breve{R}_{N}(\tau) & =\frac{1}{N-k_{s}} \sum_{k=k_{s}+1}^{N} \psi^{2}(k, \tau)
\end{aligned}
$$

$\psi(k, \tau)$ can be obtained through tedious but straightforward calculations as follows.

$$
\begin{aligned}
\psi(k, \tau) & =-\frac{\partial \breve{\varepsilon}(k, \tau)}{\partial \tau} \\
& =\boldsymbol{\varphi}_{\tau}^{T}(k, \tau) \boldsymbol{R}^{-1}(N, \tau) \boldsymbol{f}(N, \tau)+\boldsymbol{\varphi}^{T}(k, \tau) \boldsymbol{R}^{-1}(N, \tau) \boldsymbol{f}_{\tau}(N, \tau) \\
& -\boldsymbol{\varphi}^{T}(k, \tau) \boldsymbol{R}^{-1}(N, \tau)\left[\boldsymbol{R}_{\tau}(N, \tau)+\boldsymbol{R}_{\tau}^{T}(N, \tau)\right] \boldsymbol{R}^{-1}(N, \tau) \boldsymbol{f}(N, \tau)
\end{aligned}
$$

where 


$$
\begin{aligned}
\boldsymbol{R}_{\tau}(N, \tau) & =\frac{1}{N-k_{s}} \sum_{k=k_{s}+1}^{N} \boldsymbol{\varphi}_{\tau}(k, \tau) \boldsymbol{\varphi}^{T}(k, \tau) \\
\boldsymbol{f}_{\tau}(N, \tau) & =\frac{1}{N-k_{s}} \sum_{k=k_{s}+1}^{N} \boldsymbol{\varphi}_{\tau}(k, \tau) \xi_{0 \bar{y}}(k) \\
\boldsymbol{\varphi}_{\tau}(k, \tau) & =\frac{\partial \boldsymbol{\varphi}(k, \tau)}{\partial \tau} \\
& =\left[\mathbf{0}_{1 \times n},-\xi_{(n-m) \bar{u}}(k-\tilde{\tau}), \cdots,-\xi_{(n-1) \bar{u}}(k-\tilde{\tau})\right]^{T}
\end{aligned}
$$

The SEPNLS method can be summarized as follows.

(1) Let $j=0$. Set the initial estimate $\widehat{\tau}_{N}^{(0)}$ and the considerable upper bound of time delay $\bar{\tau}$.

(2) Perform the following.

(a) Compute

$$
\Delta \widehat{\tau}_{N}^{(j+1)}=-\breve{R}_{N}^{-1}\left(\widehat{\tau}_{N}^{(j)}\right) \breve{V}_{N}^{\prime}\left(\widehat{\tau}_{N}^{(j)}\right)
$$

(b) Compute

$$
\widehat{\tau}_{N}^{(j+1)}=\widehat{\tau}_{N}^{(j)}+\Delta \widehat{\tau}_{N}^{(j+1)}
$$

(c) Check if $0 \leq \widehat{\tau}_{N}^{(j+1)} \leq \bar{\tau}$. If not, let $\Delta \widehat{\tau}_{N}^{(j+1)}$ $=0.5 \Delta \widehat{\tau}_{N}^{(j+1)}$ and go back to (b).

(d) Check if $\breve{V}_{N}\left(\widehat{\tau}_{N}^{(j+1)}\right) \leq \breve{V}_{N}\left(\widehat{\tau}_{N}^{(j)}\right)$. If not, let $\Delta \widehat{\tau}_{N}^{(j+1)}=0.5 \Delta \widehat{\tau}_{N}^{(j+1)}$ and go back to (b).

(3) Terminate the algorithm if the stopping condition is satisfied. Otherwise, let $j=j+1$ and go back to step 2 .

Finally, by substituting $\widehat{\tau}_{N}$ into (16), we can estimate linear parameter vector $\boldsymbol{\theta}$ the linear LS method (16).

Remark 3: Comparative discussions on separable and unseparable algorithms can be found in the literature ${ }^{(15) \sim(17)}$. In reference (17), it is shown that the computational burden per iteration is of the same order for the separable and unseparable algorithms. In references (15) (16), it is pointed out through theoretical analysis that $\breve{R}_{N}$ in (21) is better-conditioned than $R_{N}$ in (12). Therefore the separable algorithm is likely to converge faster than the nonseparable one, especially in the ill-conditioned cases. And numerical examples confirmed this ${ }^{(15)(16)}$. In our problem, however, we found through numerical examples that at least for the SISO systems under study, the separable algorithm converges only slightly faster than the nonseparable one and hence both alternative methods are acceptable. This might be due to that there exists only one single nonlinear parameter (the time delay $\tau$ ) in the estimation problem so that the problem is considered to be relatively simple. Further comparative stduies for MIMO systems should be performed in future.

\section{Nonlinear Instrumental Variable Methods}

It is well known that the LS estimates are usually biased in the presence of measurement noise. In this section two novel nonlinear IV methods, i.e., UNSEPNIV and SEPNIV methods are proposed to obtain consistent estimates. In the problem of linear parameter estimation, the IV method is a well-known approach that eliminates the estimate bias due to measurement noise through correlation technique ${ }^{(11)}$. Here, the IV method is extended to the problem of nonlinear parameter estimation.

5.1 UNSEPNIV Method We first define the following IV vector by using the input signal $\bar{u}(k)$ and the sampled noise-free output $x(k)$.

$$
\begin{aligned}
\boldsymbol{M}(k, \boldsymbol{\Theta})=[ & -\xi_{1 \bar{x}}(k), \cdots,-\xi_{n \bar{x}}(k), \xi_{(n-m+1) \bar{u}}(k-\tilde{\tau}), \\
& \left.\cdots, \xi_{n \bar{u}}(k-\tilde{\tau}),-\sum_{i=1}^{m} b_{i} \xi_{(n-m+i-1) \bar{u}}(k-\tilde{\tau})\right]^{T}
\end{aligned}
$$

where

$$
\xi_{i \bar{x}}(k)=\frac{\left(\frac{T}{2}\right)^{i}\left(1+q^{-1}\right)^{i}\left(1-q^{-1}\right)^{n-i}}{\left[\alpha\left(1-q^{-1}\right)+\frac{T}{2}\left(1+q^{-1}\right)\right]^{n}} \bar{x}(k) \ldots
$$

and $\bar{x}(k)=\left(1+z^{-1}\right) x(k) / 2$.

Notice that $\boldsymbol{\zeta}(k, \boldsymbol{\Theta})$ defined in (14) can be expressed as

$$
\boldsymbol{\zeta}(k, \boldsymbol{\Theta})=\boldsymbol{M}(k, \boldsymbol{\Theta})+\boldsymbol{\zeta}_{v}(k)
$$

where

$$
\boldsymbol{\zeta}_{v}(k)=\left[-\xi_{1 \bar{v}}(k), \cdots,-\xi_{n \bar{v}}(k), \mathbf{0}_{1 \times(m+1)}\right]^{T} \ldots
$$

Motivated by the common IV technique for linear system identificantion, we can modify the UNSEPNLS method to the following UNSEPNIV method, by using $\boldsymbol{M}(k, \Theta)$ as the IV vector.

$$
\widehat{\boldsymbol{\Theta}}_{I V N}^{(j+1)}=\widehat{\boldsymbol{\Theta}}_{I V N}^{(j)}-\mu_{N}^{(j)}\left[R_{I V N}\left(\widehat{\boldsymbol{\Theta}}_{I V N}^{(j)}\right)\right]^{-1} V_{I V N}^{\prime}\left(\widehat{\boldsymbol{\Theta}}_{I V N}^{(j)}\right)
$$

where

$$
\begin{aligned}
& V_{I V N}^{\prime}(\boldsymbol{\Theta})=-\frac{1}{N-k_{s}} \sum_{k=k_{s}+1}^{N} \boldsymbol{M}(k, \boldsymbol{\Theta}) \epsilon(k, \boldsymbol{\Theta}) \\
& R_{I V N}(\boldsymbol{\Theta})=\frac{1}{N-k_{s}} \sum_{k=k_{s}+1}^{N} \boldsymbol{M}(k, \boldsymbol{\Theta}) \boldsymbol{\zeta}^{T}(k, \boldsymbol{\Theta})
\end{aligned}
$$

We will analyze the asymptotical property of the UNSEPNIV estimate. Keeping assumption 2 in mind and through some correlation operations, we have the following results.

$$
\begin{gathered}
\lim _{N \rightarrow \infty} R_{I V N}(\boldsymbol{\Theta})=\frac{1}{N-k_{s}} \sum_{k=k_{s}+1}^{N} \boldsymbol{M}(k, \boldsymbol{\Theta}) \boldsymbol{M}^{T}(k, \boldsymbol{\Theta}) \cdots(31) \\
\lim _{N \rightarrow \infty} V_{I V N}^{\prime}(\boldsymbol{\Theta})=-\frac{1}{N-k_{s}} \sum_{k=k_{s}+1}^{N} \boldsymbol{M}(k, \boldsymbol{\Theta})\left(\xi_{0 \bar{x}}(k)-\boldsymbol{m}^{T}(k, \tau) \boldsymbol{\theta}\right)
\end{gathered}
$$

The above results indicate that when the data length is sufficiently large, $R_{I V N}(\boldsymbol{\Theta})$ and $V_{I V N}^{\prime}(\boldsymbol{\Theta})$ converge to their counterparts of the noise-free case. Then the UNSEPNIV estimate is expected to converge to the noisefree UNSEPNLS estimate, when the data length is sufficiently large. Therefore if the noise-free UNSEPNLS 
reaches the global minimum, then UNSEPNIV estimate likely converges consistently to the true value.

Remark 4: In practice, however, the noise-free output is never known. Therefore, a boot-strap scheme is usually used to generate the instrumental variables ${ }^{(18)(19)}$. The estitmated noise-free output $\widehat{\bar{x}}(k)$ is obtained as

$$
\begin{aligned}
& \widehat{\bar{z}}(k)= \\
& \sum_{i=1}^{m} \hat{b}_{i}\left(\frac{T}{2}\right)^{n-m+i}\left(1+z^{-1}\right)^{n-m+i}\left(1-z^{-1}\right)^{m-i} \\
& \sum_{i=0}^{n} \hat{a}_{i}\left(\frac{T}{2}\right)^{i}\left(1+z^{-1}\right)^{i}\left(1-z^{-1}\right)^{n-i} \\
& \widehat{\bar{x}}(k)=\hat{\bar{z}}(k-\widehat{\widetilde{\tau}})
\end{aligned}
$$

The boot-strap approach is rather ad hoc, and strict analysis of convergency is still not available in the literature. However, empirical studies indicate the boot-strap algorithms converge quite well.

Remark 5: In practice, it is recommended to first perform several iterations of the UNSEPNLS method and then to switch to the UNSEPNIV method which is initialized by the UNSEPNLS estimate.

We should notice that the UNSEPNIV estimate does not imply that it minimizes the LS criterion. However if the IV estimate is consistent, it should minimize the mean squares of the output error:

$$
V_{I V}\left(\boldsymbol{\theta}_{I V}, \tau\right)=\frac{1}{N-k_{s}} \sum_{k=k_{s}+1}^{N}(\bar{y}(k)-\widehat{\bar{x}}(k))^{2} \ldots
$$

The UNSEPNIV method is summarized as follows.

(1) Let $j=0$. Set the initial estimate $\widehat{\boldsymbol{\Theta}}_{I V N}^{(0)}$ and the considerable upper bound of time delay $\bar{\tau}$. Generate the estimated noise-free output by using $\widehat{\Theta}_{I V N}^{(0)}$.

(2) Perform the following.

(a) Compute

$$
\Delta \widehat{\boldsymbol{\Theta}}_{I V N}^{(j+1)}=-\left[R_{I V N}\left(\widehat{\boldsymbol{\Theta}}_{I V N}^{(j+1)}\right)\right]^{-1} V_{I V N}^{\prime}\left(\widehat{\boldsymbol{\Theta}}_{I V N}^{(j+1)}\right)
$$

(b) Compute

$$
\widehat{\boldsymbol{\Theta}}_{I V N}^{(j+1)}=\widehat{\boldsymbol{\Theta}}_{I V N}^{(j)}+\Delta \widehat{\boldsymbol{\Theta}}_{I V N}^{(j+1)}
$$

(c) Check if $0 \leq \widehat{\tau}_{I V N}^{(j+1)} \leq \bar{\tau}$. If not, let $\Delta \widehat{\boldsymbol{\Theta}}_{I V N}^{(j+1)}$ $=0.5 \Delta \widehat{\boldsymbol{\Theta}}_{I V N}^{(j+1)}$ and go back to (b).

(d) Check if the model (33) that generates the estimated noise-free output is stable. If not, let $\Delta \widehat{\boldsymbol{\Theta}}_{I V N}^{(j+1)}=0.5 \Delta \widehat{\boldsymbol{\Theta}}_{I V N}^{(j+1)}$ and go back to (b).

(e) Generate the estimated noise-free output by using $\widehat{\Theta}_{I V N}^{(j+1)}$.

(f) Check if $\left|V_{I V}\left(\widehat{\boldsymbol{\Theta}}_{I V N}^{(j+1)}\right)\right| \leq\left|V_{I V}\left(\widehat{\boldsymbol{\Theta}}_{I V N}^{(j)}\right)\right|$. If not, let $\Delta \widehat{\boldsymbol{\Theta}}_{I V N}^{(j+1)}=0.5 \Delta \widehat{\boldsymbol{\Theta}}_{I V N}^{(j+1)}$ and go back to (b).

(3) Terminate the algorithm if the stopping condition is satisfied. Otherwise, let $j=j+1$ and go back to step 2 .
5.2 SEPNIV Method By the similar idea shown in the previous subsection, we can also modify the SEPNLS method to a SEPNIV method.

Define the IV vector

$$
\begin{aligned}
\boldsymbol{m}^{T}(k, \tau)=\left[-\xi_{1 \bar{x}}(k), \cdots,-\xi_{n \bar{x}}(k),\right. & \\
& \left.\xi_{(n-m+1) \bar{u}}(k-\tilde{\tau}), \cdots, \xi_{n \bar{u}}(k-\tilde{\tau})\right]
\end{aligned}
$$

Then we can estimate the linear parameters by the linear IV method as

$$
\begin{aligned}
\widehat{\boldsymbol{\theta}}_{I V N}(\tau) & =\boldsymbol{R}_{I V}^{-1}(N, \tau) \boldsymbol{f}_{I V}(N, \tau) \\
\boldsymbol{R}_{I V}(N, \tau) & =\frac{1}{N-k_{s}} \sum_{k=k_{s}+1}^{N} \boldsymbol{m}(k, \tau) \boldsymbol{\varphi}^{T}(k, \tau) \ldots \\
\boldsymbol{f}_{I V}(N, \tau) & =\frac{1}{N-k_{s}} \sum_{k=k_{s}+1}^{N} \boldsymbol{m}(k, \tau) \xi_{0 \bar{y}}(k)
\end{aligned}
$$

And the residual is given as

$$
\breve{\varepsilon}_{I V}(k, \tau)=\xi_{0 \bar{y}}(k)-\varphi^{T}(k, \tau) \boldsymbol{R}_{I V}^{-1}(N, \tau) \boldsymbol{f}_{I V}(N, \tau)
$$

Then the time delay can be obtained through the following iterative search algorithm.

$$
\widehat{\tau}_{I V N}^{(j+1)}=\widehat{\tau}_{I V N}^{(j)}-\mu_{N}^{(j)}\left[\breve{R}_{I V N}\left(\widehat{\tau}_{I V N}^{(j)}\right)\right]^{-1} \breve{V}_{I V N}^{\prime}\left(\widehat{\tau}_{I V N}^{(j)}\right)
$$

where

$$
\begin{aligned}
& \breve{V}_{I V N}^{\prime}(\tau)=-\frac{1}{N-k_{s}} \sum_{k=k_{s}+1}^{N} \psi_{I V}(k, \tau) \breve{\varepsilon}_{I V}(k, \tau) \\
& \breve{R}_{I V N}(\tau)=\frac{1}{N-k_{s}} \sum_{k=k_{s}+1}^{N} \psi_{I V}(k, \tau) \psi_{m}(k, \tau)
\end{aligned}
$$

$\psi_{m}(k, \tau)$ is a slight modification of $\psi(k, \tau)$ given in $(23)$ :

$$
\begin{aligned}
& \psi_{m}(k, \tau)= \\
& \boldsymbol{\varphi}_{\tau}^{T}(k, \tau) \boldsymbol{R}_{I V}^{-1}(N, \tau) \boldsymbol{f}_{I V}(N, \tau)+\boldsymbol{\varphi}^{T}(k, \tau) \boldsymbol{R}_{I V}^{-1}(N, \tau) \boldsymbol{f}_{\tau}(N, \tau) \\
& -\boldsymbol{\varphi}^{T}(k, \tau) \boldsymbol{R}_{I V}^{-1}(N, \tau)\left[\boldsymbol{R}_{\tau}(N, \tau)+\boldsymbol{R}_{\tau}^{T}(N, \tau)\right] \boldsymbol{R}_{I V}^{-1}(N, \tau) \boldsymbol{f}_{I V}(N, \tau)
\end{aligned}
$$

and $\psi_{I V}(k, \tau)$ is the IV to make $\breve{V}_{I V N}^{\prime}(\tau)$ and $\breve{R}_{I V N}(\tau)$ consistent:

$$
\begin{aligned}
& \psi_{I V}(k, \tau)= \\
& \boldsymbol{\varphi}_{\tau}^{T}(k, \tau) \boldsymbol{R}_{I V}^{-1}(N, \tau) \boldsymbol{f}_{I V}(N, \tau)+\boldsymbol{m}^{T}(k, \tau) \boldsymbol{R}_{I V}^{-1}(N, \tau) \boldsymbol{f}_{\tau}(N, \tau) \\
& -\boldsymbol{m}^{T}(k, \tau) \boldsymbol{R}_{I V}^{-1}(N, \tau)\left[\boldsymbol{R}_{\tau}(N, \tau)+\boldsymbol{R}_{\tau}^{T}(N, \tau)\right] \boldsymbol{R}_{I V}^{-1}(N, \tau) \boldsymbol{f}_{I V}(N, \tau)
\end{aligned}
$$

Similar to the analysis in the previous subsection, we will investigate here the asymptotical properties of $\breve{R}_{I V N}(\tau)$ and $\breve{V}_{I V N}^{\prime}(\tau)$ which influence the result of the SEPNIV estimate.

Keeping assumption 2 in mind and through some correlation operations, we first have the following results.

$$
\lim _{N \rightarrow \infty} \boldsymbol{R}_{I V}(N, \tau)=\frac{1}{N-k_{s}} \sum_{k=k_{s}+1}^{N} \boldsymbol{m}(k, \tau) \boldsymbol{m}^{T}(k, \tau)
$$




$$
\lim _{N \rightarrow \infty} \boldsymbol{f}_{I V}(N, \tau)=\frac{1}{N-k_{s}} \sum_{k=k_{s}+1}^{N} \boldsymbol{m}(k, \tau) \xi_{0 \bar{x}}(k)
$$

Therefore $\widehat{\boldsymbol{\theta}}_{I V N}(\tau)$ is consistent if a consistent estimate of $\tau$ is used in (36).

Similarly, we furthermore have

$$
\begin{array}{r}
\lim _{N \rightarrow \infty} \boldsymbol{R}_{\tau}(N, \tau)=\frac{1}{N-k_{s}} \sum_{k=k_{s}+1}^{N} \boldsymbol{\varphi}_{\tau}(k, \tau) \boldsymbol{m}^{T}(k, \tau) \\
\ldots \ldots \ldots \ldots \ldots \ldots \ldots \ldots \\
\lim _{N \rightarrow \infty} \boldsymbol{f}_{\tau}(N, \tau)=\frac{1}{N-k_{s}} \sum_{k=k_{s}+1}^{N} \boldsymbol{\varphi}_{\tau}(k, \tau) \xi_{0 \bar{x}}(k)
\end{array}
$$

From (42), (43), (44) and (45), we can conclude that $\psi_{I V}(k, \tau)$ given in $(41)$ also converges to its noise free counterpart when the data length is sufficiently large.

By using $\psi_{m}(k, \tau)$ and $\psi_{I V}(k, \tau)$ given in (40) and (41), and through some correlation operations, we have the following results.

$$
\lim _{N \rightarrow \infty} \breve{R}_{I V N}(\tau)=\lim _{N \rightarrow \infty} \frac{1}{N-k_{s}} \sum_{k=k_{s}+1}^{N} \psi_{I V}^{2}(k, \tau) \cdots
$$

$$
\begin{aligned}
& \lim _{N \rightarrow \infty} \breve{V}_{I V N}^{\prime}(\tau)= \\
& \quad-\lim _{N \rightarrow \infty} \frac{1}{N-k_{s}} \sum_{k=k_{s}+1}^{N} \psi_{I V}(k, \tau)\left(\xi_{0 \bar{x}}(k)-\boldsymbol{m}^{T}(k, \tau) \widehat{\boldsymbol{\theta}}_{I V N}(\tau)\right)
\end{aligned}
$$

The results of (46) and (47) imply that $\breve{R}_{I V N}(\tau)$ and $\breve{V}_{I V N}^{\prime}(\tau)$ do not include any bias term due to noise. Therefore $\widehat{\tau}_{I V N}$ given in (38) is also expected to converge to the noise-free SEPNLS estimate, when the data length is sufficiently large.

As mentioned in the previous subsection, in practice, however, the noise-free output is never known. Therefore, a boot-strap scheme is usually used to generate the instrumental variables. And the SEPNIV algorithm can be initialized by the SEPNLS estimate.

Also, we should notice that the SEPNIV estimate does not imply that it minimizes the LS criterion. If the IV estimate is consistent, it should minimize the mean squares output error criterion (34).

The SEPNIV method is summarized as follows.

(1) Let $j=0$. Set the initial estimate $\widehat{\boldsymbol{\theta}}_{I V N}^{0}$ and $\widehat{\tau}_{I V N}^{(0)}$, and the considerable upper bound of time delay $\bar{\tau}$. Generate the estimated noise-free output by using $\widehat{\boldsymbol{\theta}}_{I V N}^{(0)}$ and $\widehat{\tau}_{I V N}^{(0)}$.

(2) Perform the following.

(a) Compute

$$
\Delta \widehat{\tau}_{I V N}^{(j+1)}=-\left[\breve{R}_{I V N}\left(\widehat{\tau}_{I V N}^{(j)}\right)\right]^{-1} \breve{V}_{I V N}^{\prime}\left(\widehat{\tau}_{I V N}^{(j)}\right)
$$

(b) Compute

$$
\widehat{\tau}_{I V N}^{(j+1)}=\widehat{\tau}_{I V N}^{(j)}+\Delta \widehat{\tau}_{I V N}^{(j+1)}
$$

Table 1. Estimates in the case of $\mathrm{NSR}=1 \%$.

\begin{tabular}{r||r|r|r|r}
\hline & \multicolumn{1}{c|}{$\widehat{\tau}(3.13)$} & \multicolumn{1}{c|}{$\widehat{a_{1}}(6.0)$} & \multicolumn{1}{c|}{$\widehat{a_{2}}(3.0)$} & \multicolumn{1}{c}{$\widehat{b_{1}}(1.0)$} \\
\hline \hline \multirow{2}{*}{$\mathrm{A}$} & 3.1305 & 6.0308 & 3.0171 & 1.0060 \\
& \pm 0.0003 & \pm 0.0075 & \pm 0.0026 & \pm 0.0153 \\
\hline \multirow{2}{*}{$\mathrm{B}$} & 3.1305 & 6.0308 & 3.0171 & 1.0060 \\
& \pm 0.0003 & \pm 0.0075 & \pm 0.0026 & \pm 0.0153 \\
\hline \multirow{2}{*}{$\mathrm{C}$} & 3.1310 & 6.0514 & 3.0270 & 1.0093 \\
& \pm 0.0003 & \pm 0.0074 & \pm 0.0025 & \pm 0.0151 \\
\hline \multirow{2}{*}{$\mathrm{D}$} & 3.1310 & 6.0514 & 3.0270 & 1.0093 \\
& \pm 0.0003 & \pm 0.0074 & \pm 0.0025 & \pm 0.0151 \\
\hline
\end{tabular}

Table 2. Estimates in the case of $\mathrm{NSR}=5 \%$.

\begin{tabular}{r||r|r|r|r}
\hline & \multicolumn{1}{|c|}{$\widehat{\tau}(3.13)$} & \multicolumn{1}{c|}{$\widehat{a_{1}}(6.0)$} & \multicolumn{1}{c|}{$\widehat{a_{2}}(3.0)$} & \multicolumn{1}{c}{$\widehat{b_{1}(1.0)}$} \\
\hline \hline \multirow{2}{*}{$\mathrm{A}$} & 3.1202 & 5.6069 & 2.8139 & 0.9365 \\
& \pm 0.0023 & \pm 0.0878 & \pm 0.0443 & \pm 0.0149 \\
\hline \multirow{2}{*}{$\mathrm{B}$} & 3.1202 & 5.6069 & 2.8139 & 0.9365 \\
& \pm 0.0023 & \pm 0.0878 & \pm 0.0443 & \pm 0.0149 \\
\hline \multirow{2}{*}{$\mathrm{C}$} & 3.1309 & 6.0491 & 3.0256 & 1.0090 \\
& \pm 0.0016 & \pm 0.0754 & \pm 0.0369 & \pm 0.0126 \\
\hline \multirow{2}{*}{$\mathrm{D}$} & 3.1309 & 6.0491 & 3.0256 & 1.0090 \\
& \pm 0.0016 & \pm 0.0754 & \pm 0.0369 & \pm 0.0126 \\
\hline
\end{tabular}

Table 3. Estimates in the case of NSR $=10 \%$.

\begin{tabular}{r||r|r|r|r}
\hline & \multicolumn{1}{c|}{$\widehat{\tau}(3.13)$} & \multicolumn{1}{c|}{$\widehat{a_{1}}(6.0)$} & $\widehat{a_{2}}(3.0)$ & \multicolumn{1}{c}{$\widehat{b}_{1}(1.0)$} \\
\hline \hline \multirow{2}{*}{$\mathrm{A}$} & $\begin{array}{r}3.0963 \\
\pm 0.0047\end{array}$ & $\begin{array}{r}4.7914 \\
\pm 0.1348\end{array}$ & $\begin{array}{r}2.4289 \\
\pm 0.0666\end{array}$ & $\begin{array}{r}0.8040 \\
\pm 0.0225\end{array}$ \\
\hline \multirow{2}{*}{$\mathrm{B}$} & 3.0963 & 4.7915 & 2.4290 & 0.8040 \\
& \pm 0.0049 & \pm 0.1407 & \pm 0.0714 & \pm 0.0237 \\
\hline \multirow{2}{*}{$\mathrm{C}$} & 3.1307 & 6.0475 & 3.0245 & 1.0088 \\
& \pm 0.0032 & \pm 0.1494 & \pm 0.0729 & \pm 0.0251 \\
\hline \multirow{2}{*}{$\mathrm{D}$} & 3.1307 & 6.0475 & 3.0245 & 1.0088 \\
& \pm 0.0032 & \pm 0.1494 & \pm 0.0729 & \pm 0.0251 \\
\hline
\end{tabular}

(c) Check if $0 \leq \widehat{\tau}_{I V N}^{(j+1)} \leq \bar{\tau}$. If not, let $\Delta \widehat{\tau}_{I V N}^{(j+1)}=$ $0.5 \Delta \widehat{\tau}_{I V N}^{(j+1)}$ and go back to (b).

(d) Compute

$$
\widehat{\boldsymbol{\theta}}_{I V N}^{(j+1)}=\boldsymbol{R}_{I V}^{-1}\left(N, \widehat{\tau}^{(j+1)}\right) \boldsymbol{f}_{I V}\left(N, \widehat{\tau}^{(j+1)}\right) .
$$

(e) Check if the model (33) that generates the estimated noise-free output is stable. If not, let $\Delta \widehat{\tau}_{I V N}^{(j+1)}=0.5 \Delta \widehat{\tau}_{I V N}^{(j+1)}$ and go back to (b).

(f) Generate the estimated noise-free output by using $\widehat{\boldsymbol{\theta}}_{I V N}^{(j+1)}$ and $\widehat{\tau}_{I V N}^{(j+1)}$.

(g) Check if $\left|V_{I V}\left(\widehat{\boldsymbol{\theta}}_{I V N}^{(j+1)}, \widehat{\tau}_{I V N}^{(j+1)}\right)\right| \leq\left|V_{I V}\left(\widehat{\boldsymbol{\theta}}_{I V N}^{(j)}, \widehat{\tau}_{I V N}^{(j)}\right)\right|$. If not, let $\Delta \widehat{\tau}_{I V N}^{(j+1)}=0.5 \Delta \widehat{\tau}_{I V N}^{(j+1)}$ and go back to (b).

(3) Terminate the algorithm if the stopping condition is satisfied. Otherwise, let $j=j+1$ and go back to step 2 .

\section{Numerical Results}

Consider the following SISO continuous-time system:

$$
\begin{aligned}
& \ddot{x}(t)+a_{1} \dot{x}(t)+a_{2} x(t)=b_{1} u(t-\tau) \\
& a_{1}=6.0, \quad a_{2}=3.0, \quad b_{1}=1.0, \quad \tau=3.130
\end{aligned}
$$

Notice that many practical systems such as thermal processes, chemical processes and biological systems can be described by a first or second-order linear system with time delay.

The input signal is output of a zero-order hold driven by a white signal filtered by a second-order Butterworth filter

$$
L(p)=\frac{1}{\left(p / \omega_{c}\right)^{2}+\sqrt{2}\left(p / \omega_{c}\right)+1} \quad\left(\omega_{c}=4.0\right) \ldots
$$




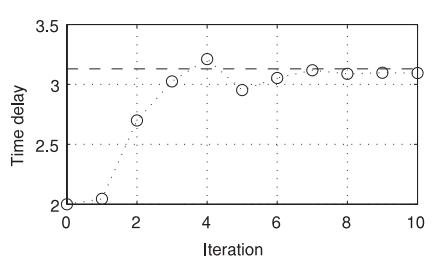

(A) UNSEPNLS method

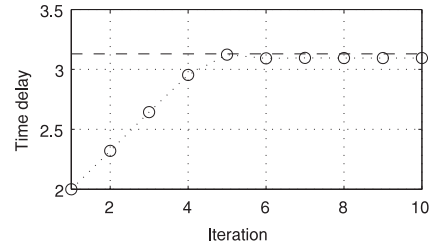

(B) SEPNLS method

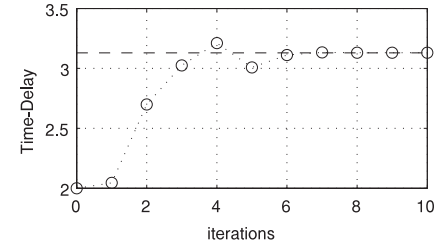

(C) UNSEPNIV method

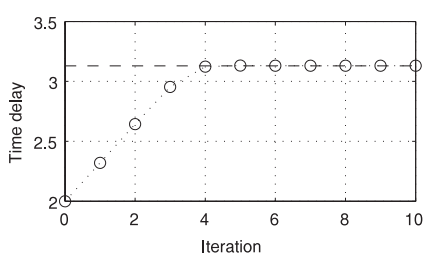

(D) SEPNIV method

Fig. 1. Convergency behaviour of the time delay estimate (NSR $=10 \%$ ).

which is discretized by the bilinear transformation.

The input and output signals are sampled with a sampling period taken as $T=0.05$, and $\alpha$ in the lowpass pre-filter $Q(p)$ is 0.4 . Experiments are carried out through 20 realizations of the measurement noise and random input signal, when NSR (noise to signal ratio, defined as the ratio of the noise standard deviation versus the noise-free output standard deviation) is 1\%, $5 \%$ and $10 \%$ respectively with a data length of 4000 . All the algorithms are implemented for 10 iterations, whereas for the IV algorithms, the first 2 iterations are performed by the LS methods, as suggested in remark 5 .

For the UNSEPNLS method, the initial values are set as $\left[\widehat{\theta}^{T(0)}, \widehat{\tau}^{(0)}\right]^{T}=[1,1,1,2.0]^{T}$. And for the SEPNLS method, the initial value is set as $\widehat{\tau}^{(0)}=2.0$. Notice that the iteratative identification algorithms are basically based on gradient-following technique. And it is well known that the estimates by such algorithms may be stuck at local minima. Therefore, the results may be sensitive to the initial values. For the SISO systems under study however, since there exists only one single nonlinear parameter (the time delay $\tau$ ) in the estimation problem, the problem of initial setting is considered to be relatively simple. Several trials are enough. For MIMO systems with multiple time delays, the problem is much more difficult. And the algorithms proposed here should be enhanced by some globally optimizing techniques. This issue is presently under investigation and the results will be reported elsewhere in future.

The results are shown in Tables $1 \sim 3$, where A, B, C, D denote respectively UNSEPNLS, SEPNLS, UNSEPNIV and SEPNIV methods. The tables include the mean and standard deviation of the estimates. It can be verified that the numerical results reflect the theoretical discussions quite well. The UNSEPNLS and SEPNLS methods yield almostly identical estimates, and so do the UNSEPNIV and SEPNIV methods. This confirms the results of Theorem 1 . However, it can be seen that by the LS methods, the estimates are biased when the measurement noise is not trivial. And the bias becomes greater when the noise is higher. On the other hand, UNSEPNIV and SEPNIV methods yield consistent estimates in each case, as claimed in the previous section.

The examples of the convergency behaviour of the estimate of the time delay are shown in Figure 1. As mentioned in remark 3, the unseparable algorithms may converge in a slightly slower manner than the separable algorithms. However, both approaches are acceptable since the final estimates are almostly identical as shown in Tables $1 \sim 3$.
In practice, the separable algorithms might be recommended, since they may achieve better convergency properties, although the derivations of the algorithms are more complicated. In the case of low measurement noise, the SEPNLS estimate is acceptable. When the noise effects are not negligible and assumption 2 is satisfied, we can further perform the the SEPNIV method which is initialized by the SEPNLS estimate, as suggested in remark 5 .

\section{Conclusions}

In this paper, several iterative identification algorithms for continuous-time systems with unknown time delay have been proposed. Both the UNSEPNLS and SEPNLS methods were derived. Furthermore, the UNSEPNIV and SEPNIV methods were proposed to eliminate estimate bias due to measurement noise. Simulational results showed that the UNSEPNLS and SEPNLS methods are efficient only in the case of low measurement noise, whereas the UNSEPNIV and SEPNIV methods yield consistent estimates in the presence of considerably high noise. It is believed that the proposed identification algorithms are applicable to some chemical or biological processes, where the step input test to estimate the time delay is not allowed.

(Manuscript received Nov. 24, 2003, revised May 21, 2004)

\section{References}

(1) W.X. Zheng and C.B. feng: "Identification of stochastic time lag systems in the presence of colored noise", Automatica, 26, pp.769-779 (1990)

(2) W.X. Zheng and C.B. Feng: "Optimizing search-based identification of stochastic time-delay systems", Int. J. Syst. Science, 22, pp.783-792 (1991)

(3) G. Ferretti, C. Maffezzoni, and R. Scattolini: "Recursive estimation of time delay in sampled systems", Automatica, 27, pp.653-661 (1991)

(4) H. Unbehauen and G.P. Rao: "Continuous-time approaches to system identification-a survey", Automatica, 26, pp.23-35 (1990)

(5) P.J. Gawthrop and M.T. NihtilÄ: "Identification of timedelays using a polynomial identification method", Syst. Control Lett., 5, pp.267-271 (1985)

(6) M. Agarwal and C. Canudas: "On-line estimation of time delay and continuous-time process parameters", Int. J. Control, 46, pp.295-311 (1987)

( 7 ) E.W. Bai and D.H. Chyung: "Improving delay estimates derived from least-square algorithms and padé approximations", Int. J. Syst. Science, 24, pp.745-756 (1993)

(8) P.J. Gawthrop, M.T. Nihtilä, and A. Besharati-Rad: "Recursive parameter estimation of continuous-time systems with unknown time delay", Control-Theory and Advanced Technology, 5, pp.227-248 (1989) 
(9) A.E. Pearson and C.Y. Wuu: "Decoupled delay estimation in the identification of differential delay systems", Automatica, 20, pp.761-772 (1984)

(10) J. Tuch, A. Feuer, and Z.J. Palmor: "Time delay estimation in continuous linear time-invariant systems", I.E.E.E. Trans. on Automatic Control, 39, pp.823-827 (1994)

(11) L. Ljung: "System identification-theory for the user", (Prentice Hall, 1992)

(12) P. Young: "Parameter estimation for continuous-time modelsa survey", Automatica, 17, pp. 23-39 (1981)

(13) Z.H. Jiang and W. Schaufelberger: "Block-pulse function and their applications in control systems", (Springer-Verlag, berlin, 1992)

(14) S. Sagara, Z.J. Yang, K. Wada, and T. Tsuji: "Parameter identification and adaptive control of continuous systems with zero-order hold", IFAC World Congress (1993)

(15) L.S.H. Ngia: "Separable nonlinear least-squares methods for efficient off-line and on-line modeling of systems using Kautz and Laguerre filters", I.E.E.E. Trans. on Circuits and Systems II, 48, pp.562-579 (2001)

(16) J. Sjoberg and M. Viberg: "Separable non-linear least-squares minimization-possible improvements for neural net fitting", Proceeding of IEEE Workshop in Neural Networks for Signal Processing (1997)

(17) A. Ruhe and P.A. Wedin: "Algorithms for separable nonlinear least squares problems", SIAM Rev., 22, pp.318-337 (1980)

(18) P. Young and A. Jakeman: "Refined instrumental variable methods of recursive time-series analysis. Part I. Single input, single output systems", Int. J. Control, 29, pp.1-30 (1979)

(19) P. Young and A. Jakeman: "Refined instrumental variable methods of recursive time-series analysis. Part III. Extensions", Int. J. Control, 31, pp.741-764 (1980)

Zi-Jiang Yang (Member) was born in 1964. He received

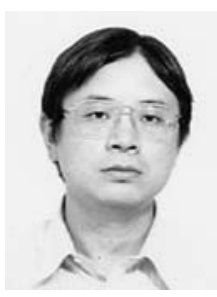
his Dr. of Eng. degree in 1992 from Kyushu University. From 1996 to 2000, he was an Associate Professor in the Faculty of Computer Engineering and System Science, Kyushu Institute of Technology. He is currently an Associate Professor in the Department of Electrical and Electronic Systems Engineering, Graduate School of Information Science and Electrical Engineering, Kyushu University. His research interests include system identification, and related signal processing, soft computing and motion control, etc.
Hideto Iemura (Non-member) was born in 1980. Now he is studying at Department of Electrical and Electronic System Engineering of Graduated school of Information Science and Electrical Engineering, Kyushu University. He is engaged in research on identification of time delay systems.

Shunshoku Kanae (Non-member) was born in 1961. He

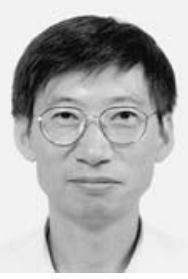
received the Dr. of Eng. degree from Kyushu University in 1995. From 1995 to 1998, he was a Research Associate at Kyushu Institute of Technology, Since October 1998, he has been a Research Associate of the Graduate School of Information Science and Electrical Engineering at Kyushu University. His research interests include system identification, mechatronics system control and soft computing, etc.

Kiyoshi Wada (Member) was born in 1947. He received

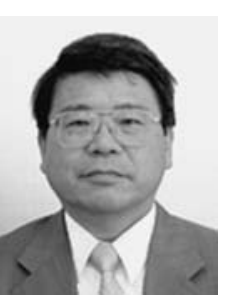
the B. E., M. E. and D. E. degrees in electrical engineering from Kyushu University, Fukuoka, Japan in 1970, 1972 and 1978 respectively. $\mathrm{He}$ is currently a professor in the department of electrical and electronic systems engineering, graduate school of information science and electrical engineering, Kyushu University. His research interests are in the areas of system identification, digital signal processing and adaptive control, etc. He is a member of the Institue of Systems, Control and Information Engineers; Society of Instrument and Control Engineers; IEEE. 